\title{
Integration Testing of Software Product Lines Using Compositional Symbolic Execution
}

\author{
Jiangfan Shi, Myra B. Cohen, and Matthew B. Dwyer \\ Department of Computer Science \& Engineering, University of Nebraska-Lincoln, \\ Lincoln, Nebraska, USA
}

\begin{abstract}
Software product lines are families of products defined by feature commonality and variability, with a well-managed asset base. Recent work in testing of software product lines has exploited similarities across development phases to reuse shared assets and reduce test effort. The use of feature dependence graphs has also been employed to reduce testing effort, but little work has focused on code level analysis of dataflow between features. In this paper we present a compositional symbolic execution technique that works in concert with a feature dependence graph to extract the set of possible interaction trees in a product family. It composes these to incrementally and symbolically analyze feature interactions. We experiment with two product lines and determine that our technique can reduce the overall number of interactions that must be considered during testing, and requires less time to run than a traditional symbolic execution technique.
\end{abstract}

\section{Introduction}

Software product line (SPL) engineering is a methodology for developing families of software programs through the managed reuse of a common and variable set of assets [18]. Variability at the application level is expressed in terms of features (functional units) that are included or excluded from the individual programs. The result is a set of similar, but unique program instantiations; in a mobile phone product line, features such as the display drivers, messaging capabilities, network support and video can be combined in different ways on top of the core features found in all phones (e.g. phone dial). While uniqueness arises from the different combination of variable features in each program, similarity comes from both the commonality found in all instantiations, as well as from matching subsets of features (i.e. partial products) between programs.

Variability, and the ability to generate many products from a core set of features, provides flexibility and enables reuse during development, but causes problems for validation. Although individual features may be validated and tested in multiple programs within the product line, this does not guarantee that specific combinations of features will work properly when composed. Research has shown that some faults - termed interaction faults - only occur under specific combinations of features [2,13] and several SPL testing techniques have attempted to account for this. For instance, Bertolino et al. [1] and Geppert et al. 6] propose 
a specification based technique to concretize a parameterized use case (based on variability), but this is an exhaustive approach that tests each product individually. This is a limitation, since the variability space grows exponentially with the number of features. If there are 4 choices for each of 10 features, then more than one million instantiations of the SPL would need to be tested to cover all possible combinations.

Kim et al. [11 use a dependency analysis to determine which features are relevant for each test within a test suite, reducing the number of products tested per test case. This technique does not consider coverage of the entire feature model, nor does it target the specific interactions; it only reduces the per-test number products. A study by Reisner et al. [20] shows that in some configurable systems - SPLs can be viewed as a type of configurable software system - analysis of control flow can reduce the possible set of configuration options that should be tested together. They do not consider other types of dependencies such as data flow, nor do they apply their approach to product lines. And neither of these studies targets specific interactions for test generation; they only reduce the number of feature combinations that should not be tested together.

In our earlier research [3], we proposed a mapping between the variability space of an SPL and a relational model in order to leverage ideas from combinatorial interaction testing (CIT), a model-based sampling technique that guarantees to test all pairs or $t$-way combinations of features within the product line. Instead of testing all program instantiations in the example above, we can test all pairs of features with approximately 24 SPL instances, or all triples of features with around 130, using a common CIT generation tool 2. Since empirical evidence suggests that lower order interactions are responsible for most interaction faults this provides some justification for CIT sampling [13].

While CIT provides a notion of coverage of the variability space, it also suffers from limitations. First, there is an expectation that all possible programs in the product line can be composed. But there may be features or groups of features that are not developed until later phases of the SPL lifetime. Second, since CIT operates at the feature combination level there is no guarantee that testing of an instance will execute the interacting code; this will depend on the quality of the test. Finally, CIT does not consider the direction of the interactions in its model, yet at the code level, interactions may happen between features in different directions. For instance, it is likely that data flows in both directions between a multi-media messaging feature of a phone and its video feature. If we have three features $\left(f_{1}, f_{2}, f_{3}\right)$, there are six directed 2 -way possible interactions.

When testing a software product line to uncover interactions, we should test from a perspective that avoids these limitations. Uncovering interactions during integration testing - where features are composed as partial products - appears to make sense from a combinatorial sense. We can test only the interactions themselves and combine products in a way that avoids redundancy. Uzuncaova et al. 24] use this idea by reusing a partial product's integration test results to generate a smaller test suite for a larger partial product. And Reis et al. [19] apply integration testing over an SPL at the specification level to avoid 
redundantly testing common partial products. Finally, Stricker et al. 23] present the ScenTED-DF methodology which uses dataflow between products to drive integration testing at the model level.

In this paper, we present a new method of analyzing software product lines for test generation. It uses ideas from CIT to drive coverage of feature interaction tuples, reduces the variability space through the use of a code-based dependency analysis, and uses directed symbolic execution to analyze possible feature combinations. The result is a method that generates constraints for all partial products at a lower cost than a full symbolic execution of an SPL code base. We also find, that by counting directed interactions, we have a more precise model of what should be tested. Finally, if we consider the constraints arising from symbolic execution, these can be used to inform a test generation technique to focus on the parts of the system that may have faults. The contributions of this work are: (1) a dataflow informed compositional symbolic integration testing method for SPLs; (2) the first discussion of interaction testing that incorporates directions; and (3) a feasibility study that shows we can reduce the number of interactions to test, and that the compositional technique uses less time than traditional symbolic execution.

\section{Background}

Software product lines are families of software systems designed for a specific domain, with a managed set of assets and well defined variability model 18 . The products all share some commonality, but are customized by variable elements of the system. Product lines vary in when they are configured. Some may be configured by the developer at build time, others allow changes through recompilation, while some use run-time constructs to change during execution.

A key artifact of a software product line is the feature (or variability) model. This is one differentiator from a general configurable system. There are many formalisms that have been developed to represent these. In this paper we use the Orthogonal Variability Model (OVM) developed by Pohl et al. [18. In OVM Variation points (VP) are shown as triangles and variants (v) are shown as rectangles. Variants will map directly to features in this paper. Dependencies are shown as solid lines (mandatory) or dashed (optional). Alternative choices are shown with arcs which are annotated with the the minimum and maximum cardinality of that VP. When there is no annotation, exactly one variant can be selected for the variation point. Additional constraints are allowed between parts of the model in the form of excludes or requires.

\subsection{Symbolic Execution}

Symbolic execution 12 is a path-sensitive program analysis technique that computes program output values as expressions over symbolic input values and constants. Symbolic execution of the code fragment: 


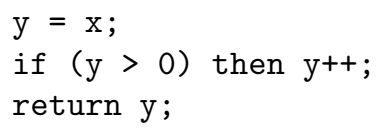

would use a symbolic value $X$ to denote the value of variable $\mathrm{x}$ on entry to the fragment. Symbolic execution determines that there are two possible paths (1) when $X>0$ the value $X+1$ is returned and (2) when $(X>0)$ the value $X$ is returned. The analysis represents the behavior of the fragment as the pairs $(X>$ $0, R E T U R N==X+1)$ and $(!(X>0), R E T U R N==X)$. The first element of a pair encodes the conjunction of constraints along an execution path - the path condition. The second element defines the values of the locations that are written along the path in terms of the symbolic input variables, e.g. $R E T U R N==X$ means that the original value for $\mathrm{x}$ is returned.

The state of a symbolic execution is a triple $(l, p c, s)$ where $l$, the current location, records the next statement to be executed, $p c$, the path condition, is the conjunction of branch conditions encoded as constraints along the current execution path, and $s: M \times \operatorname{expr}$ is a map that records a symbolic expression for each memory location, $M$, accessed along the path.

Computation statements, $m_{1}=m_{2} \odot m_{3}$, where the $m_{i} \in M$ and $\odot$ is some operator, when executed symbolically in state $(l, p c, s)$ produce a new state $\left(l+1, p c, s^{\prime}\right)$ where $\forall m \in M-\left\{m_{1}\right\}: s^{\prime}(m)=s(m)$ and $s\left(m_{1}\right)=s\left(m_{2}\right) \odot$ $s\left(m_{3}\right)$. Branching statements, if $m_{1} \odot m_{2}$ goto $d$, when executed symbolically in state $(l, p c, s)$ branch the symbolic execution to two new states $\left(d, p c \wedge\left(s\left(m_{1}\right) \odot\right.\right.$ $\left.\left.s\left(m_{2}\right)\right), s\right)$ and $\left(l+1, p c \wedge \neg\left(s\left(m_{1}\right) \odot s\left(m_{2}\right)\right), s\right)$ corresponding to the "true" and "false" evaluation of the branch condition, respectively.

An automated decision procedure is used to check the satisfiability of the updated path conditions and, when a path condition is found to be unsatisfiable, symbolic execution along that path halts. Decision procedures for a range of theories used to express path conditions, such as, linear arithmetic, arrays, and bit-vectors are available, e.g., Z3 [5].

\subsection{Symbolic Method Summary}

Several researchers [8, 17] have explored the use of method summarization in symbolic execution. In [8] summarization is used as a mechanism for optimizing the performance of symbolic execution whereas [17] explores the use of summarization as a means of abstracting program behavior to avoid symbolic execution. We adopt the definition of method summary in [17, but we forgo their use of over-approximation.

The building block for a method summary is the representation of a single execution path through method, $m$, encoded as the pair $(p c, w)$. This pair provides information about the externally visible state of the program that is relevant to an execution of $m$ at the point where $m$ returns to its caller. As described above, the $p c$ encodes the path condition and $w$ is the projection of $s$ onto the set of memory locations that are written along the executed path. We can view $w$ a conjunction of equality constraints between names of memory locations and symbolic expressions or, equivalently, as a map from locations to expressions. 

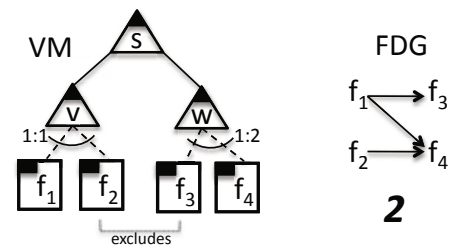

$$
\boldsymbol{8} \cdot \text { Report faults }
$$

2

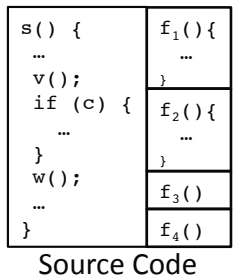

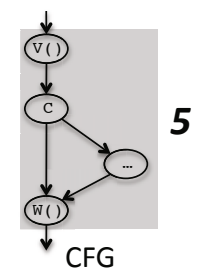

Fig. 1. Conceptual Overview of Compositional SPL Analysis

Definition 1 (Symbolic Summary [17]). A symbolic summary, for a method $m$, is a set pairs $m_{\text {sum }}: \mathcal{P}(P C \times S)$ where

$$
\forall(p c, w) \in m_{\text {sum }}: \forall\left(p c^{\prime}, w^{\prime}\right) \in m_{\text {sum }}-\{(p c, w)\}: p c \wedge p c^{\prime} \text { is unsatisfiable. }
$$

Unfortunately, it is not always possible to calculate a summary that completely accounts for the behavior of all methods. For example, methods that iterate over input data structures that are unconstrained cannot be analyzed effectively - since the length of paths are not known. We address this using the standard technique of bounding the length of paths that are analyzed.

\section{Dependence-Driven Compositional Analysis}

Our technique exploits an SPL's variability model and the inter-dependence of feature implementations to reduce the cost of applying symbolic execution to reason about feature interactions. Figure 1 provides a conceptual overview.

As explained in Section 1 an SPL is comprised of a source code base and an OVM. The OVM and its constraints (e.g., the excludes between $f_{2}$ and $f_{3}$ ) defines the set of features that may be present in an instance of the SPL.

Our technique begins (step are denoted by large bold italic numerals in the figure) by applying standard control flow and dependence analyses on the code base. The former results in a control flow graph (CFG) and the latter results in a program dependence graph (PDG). In step 2, the PDG is analyzed to calculate a feature dependence graph (FDG) which reflects inter-feature dependences. The edges of the FDG are pruned to be consistent with the OVM, e.g., the edge from $f_{2}$ to $f_{3}$ is not present.

Step 3 involves the calculation, from the FDG, of the hierarchy of all $k$-way feature interaction trees. The structure of this hierarchy reflects how lower-order interactions can be composed to create higher-order interactions. For instance, 
how the interaction among $f_{1}, f_{2}$, and $f_{4}$ can be constructed by combining $f_{1}$ with an existing interaction for $f_{2}$ and $f_{4}$.

The interaction tree hierarchy is used to guide the calculation of symbolic summaries for all interaction trees in a compositional fashion. This begins, in Step 4, by applying symbolic execution to the source code of the individual features in isolation. When composing two existing summaries, for example $f_{1}$ and $f_{3}$, to create a 2-way interaction tree, a summary of the behavior of the common SPL code which leads between those summaries must be calculated. Step 5 achieves this by locating the calls to the features in the CFG and calculating a chop [21] - shown as the shaded figure in the CFG - the edges of the chop are used to guide a customized symbolic execution to produce an edge summary. In step 6, a pair of existing lower-order interaction summaries and the edge summary are composed to produce a higher-order summary - such a summary is illustrated at point 7 in the figure.

In step 8, summaries can be exploited to detect faults, via comparison to fault oracles, or to generate tests by solving the constraints generated by symbolic execution and composition. We describe the major elements next.

\subsection{Relating SPL Models to Implementations}

An SPL implementation can be partitioned into regions of code that implement each feature; the remaining code implements the common functionality shared by all SPL instances. There are many implementation mechanisms for realizing variability in a code base [10. Our methodology can target these by adapting the summary computation for Step 4 and feature dependence graph construction for Step 2, but for simplicity it suffices to view features as methods where common code makes calls on those methods.

In the remainder of this section, we assume the existence of a mapping from in the OVM to methods in a code base; we use the name of a feature to denote the method when no confusion will arise. Features can be called from multiple points in the common code, but to simplify the presentation of our technique, we assume each feature is called from a single call site.

Given a pair of features, $f_{1}$ and $f_{2}$, where the call to $f_{2}$ is reachable in the CFG from the call to $f_{1}$, their common region is the source code chop [21] arising when the calls are used as the chop criterion. This chop is a single-entry singleexit sub-graph of the program control flow graph (CFG) where the entry node is the call to $f_{1}$ and the exit node is the call to $f_{2}$. The CFG paths within the chop overapproximate the set of feasible program executions that can lead from the return of $f_{1}$ to the call to $f_{2}$. These chops play an important role in accounting for the composite behavior of features as mediated by common code.

\subsection{Calculating Feature Interactions}

We leverage the concept of program dependences, and the PDG [16], to determine inter-feature dependences. A PDG is a directed graph, $\left(S, E_{P D G}\right)$, whose vertices are program statements, $S$, and $\left(s_{i}, s_{j}\right) \in E_{P D G}$ if $s_{i}$ defines the value 


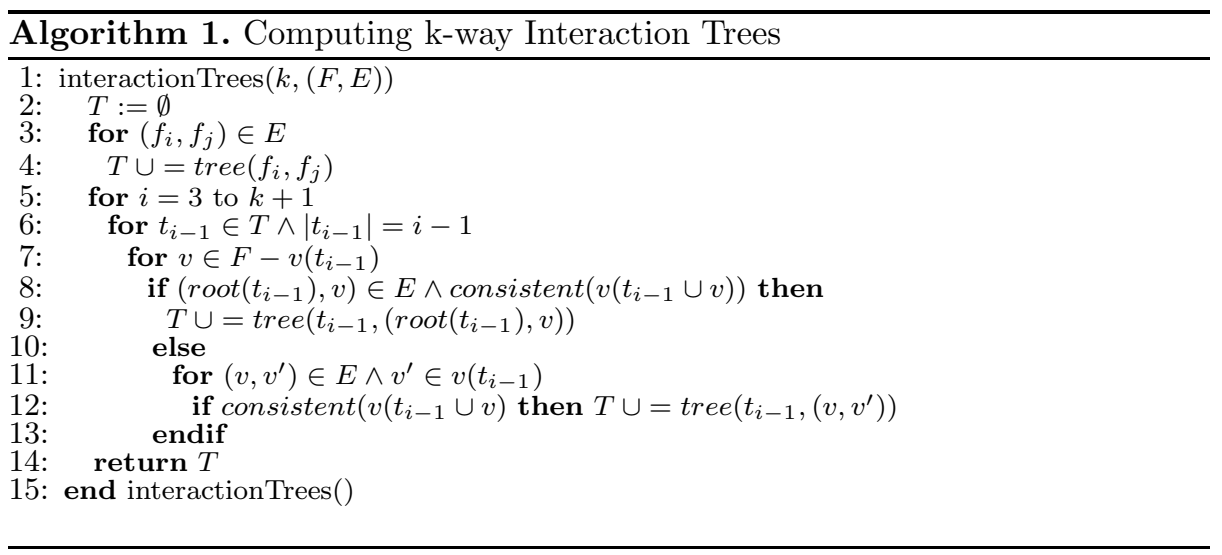

of a location that is subsequently read at $s_{j}$. A feature dependence graph (FDG) is an abstraction of the PDG for an SPL implementation.

Definition 2 (Feature Dependence Graph). Given a $P D G$ for an $S P L$, $\left(S, E_{P D G}\right)$, the FDG, $\left(F, E_{F D G}\right)$, is a directed graph whose vertices are features, $F$, and $\left(f_{i}, f_{j}\right) \in E_{F D G}$ iff $\exists s_{i}, s_{j} \in S: s_{i} \in S\left(f_{i}\right) \wedge s_{j} \in S\left(f_{j}\right) \wedge\left(s_{i}, s_{j}\right) \in E_{P D G}$ where $S(f)$ is the set of statements in feature $f$.

We capture the interaction among features by defining a tree that is embedded in the FDG. The intuition is that the root is the sink of a set of feature dependence edges. The output values of that root feature reflect the final interaction effects, and are defined in terms of the input values of the features that form the leaves of the tree.

Definition 3 (Interaction Tree). Given an FDG, $\left(F, E_{F D G}\right)$, a k-way interaction tree is an acyclic, connected, simple subgraph, $\left(F^{\prime}, E^{\prime}\right)$, where $F^{\prime} \subseteq F$, $E^{\prime} \subseteq E_{F D G},\left|F^{\prime}\right|=k$, and where $\exists r \in F^{\prime}: \forall v \in F^{\prime}: r \in v .\left(E^{\prime}\right)^{*}$. We call the common reachable vertex the root of the interaction tree.

The set of all $k$-way interaction trees for an SPL can be constructed as shown in Algorithm 1, The algorithm uses a constructor tree() which, optionally, takes an existing tree and adds edges to it expanding the set of vertices as appropriate. For a tree, $t$, the set of vertices is $v(t)$ and the root is $\operatorname{root}(t)$. Before adding a tree, the set of features in the tree must be checked to ensure they are consistent with the OVM; this is done using the predicate consistent().

The algorithm accepts $k$ and an FDG and returns the set of $k$-way interactions. It builds the set of interactions incrementally. For an $i$-way interaction, it extends an $i-1$-way interaction by adding a single additional vertex and an edge. While other strategies for building interaction trees are possible, this approach has the advantage of efficiency and simplicity. Based on our case studies, reported in Section 4, this approach is sufficient to enable significant improvement over more standard analyses of an SPL code base.

Interaction trees can be organized hierarchically based on their structure. 
Definition 4 (Interaction Hierarchy). Given a $k$-way interaction tree, $t_{k}=$ $(F, E)$, where $k>1$, we can define a pair of interaction trees $t_{i}=\left(F_{i}, E_{i}\right)$ and $t_{j}=\left(F_{j}, E_{j}\right)$, such that $F_{i} \cap F_{j}=\emptyset,\left|F_{i}\right|+\left|F_{j}\right|=k$, and $\exists\left(f_{i}, f_{j}\right) \in E$. We say that $t_{k}$ is the parent of $t_{i}$ and $t_{j}$ and, that $t_{i}$ and $t_{j}$ are the children of $t_{k}$.

The base case of the hierarchy, where $k=1$, is simply each feature in isolation. There are many ways to construct such an interaction hierarchy, since for any given k-way interaction tree cutting a single edge partitions the tree into two children. As discussed below, the hierarchy resulting from Algorithm 1 enjoys a structure that can be exploited in generating summaries of interaction pattern behavior. The parent (child) relationships among interaction trees can be recorded at the point where the tree() constructor calls are made in Algorithm1.

\subsection{Composing Feature Summaries}

Our goal is to analyze program paths that span sets of features in an SPL to support fault detection and test generation. Our approach to feature summarization involves two distinct phases: (1) the application of bounded symbolic execution to feature implementations in isolation to produce feature summaries, and (2) the matching and combination of feature summaries to produce summaries of the behavior of interaction patterns.

Phase (1) is performed by applying traditional symbolic execution where the length of the longest branch sequence is bounded to $d$ - the depth. For each feature, $f$, this results in a summary, $f_{\text {sum }}$, as defined in Section 2.

When performing symbolic execution of $f$ there are three possible outcomes: (a) a complete execution of $f$ which returns normally as analyzed within $d$ branches, (b) an exception, including assertion violations, is detected before $d$ branches are explored, and (c) the depth bound is reached. In our work, we only accumulate the outcomes falling into (a) into $f_{\text {sum }}$.

Case (b) is interesting, because it may indicate a fault in feature $f$. The isolated symbolic execution of $f$ allows for any possible state on entry to the feature, however, it is possible that a detected exception is infeasible in the context of a system execution. In future work, we will preserving results from case (b) and attempt to determine their feasibility when composed in interaction patterns with other features - this would reduce and, when interaction patterns are sufficiently large, eliminate false reports of exceptions.

For phase (2) we exploit the structure of the interaction hierarchy resulting from the application of Algorithm 1 to generate a summary for a $k$-way interaction. As discussed above, such an interaction has (potentially several) pairs of children. It suffices to select any of those pairs.

Within each pair there is a $k-1$-way interaction, $i$, which we assume has a summary $i_{\text {sum }}=\left(p c_{i}, w_{i}\right)$, and single feature, $f$, summarized as $f_{\text {sum }}=\left(p c_{f}, w_{f}\right)$, which is connected by a single edge connected to either $\operatorname{root}(i)$ or one of $i$ 's leaves, $l$. To compose $i_{\text {sum }}$ and $f_{\text {sum }}$ we must characterize the behavior of the FDG edge.

The existence of an edge $\left(f, f^{\prime}\right)$ means that there is a common region beginning at the return from $f$ and ending at the call to $f^{\prime}$. Calculating the chop that 


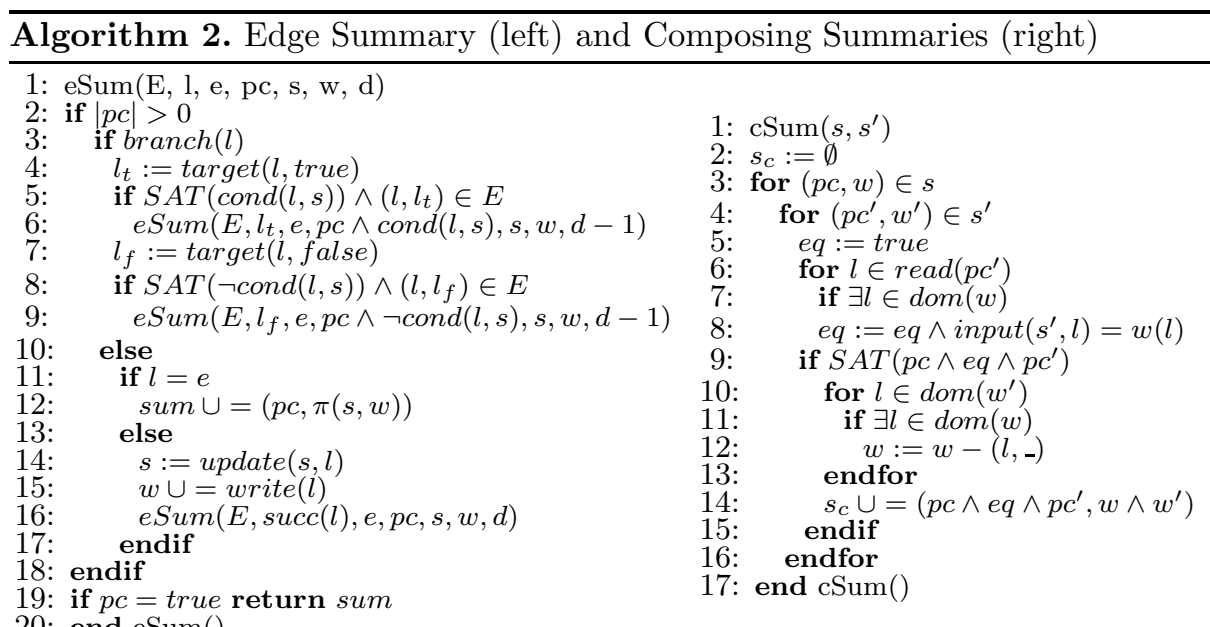

circumscribes the CFG for this region allows us to label branch outcomes that lie within the chop and to direct the symbolic execution along paths from $f$ that reach $f^{\prime}$.

Algorithm 2(left) defines this approach to calculating edge summaries. It consists of a customized depth-bounded symbolic execution that only explores a branch if that branch lies within the chop for the common region. The algorithm makes use of several helper functions. Functions determine whether an instruction is a branch, branch(), the target of a branch, target(), and the symbolic expression for a branch given a symbolic state, cond(). Functions to calculate the successor of an instruction, succ(), the set of locations written by an instruction, write(), and updating the symbolic state based on an instruction, update(), are also used. The $S A T($ ) predicate determines whether a logical formula is satisfiable. Finally, the $\pi()$ function projects a symbolic state onto a set of locations.

$\operatorname{eSum}\left(E_{\text {chop }}, \operatorname{succ}(f), f^{\prime}\right.$, true $\left., \emptyset, \emptyset, d\right)$ returns the symbolic summary for edge $\left(f, f^{\prime}\right)$ where the parameters are as follows. $E_{\text {chop }}$ is the set of edges in the CFG chop bounded by the return of $f$ and the call to $f^{\prime}, \operatorname{succ}(f)$ is the location at which initiate symbolic execution and $f^{\prime}$ is the call that terminates symbolic execution. true is the initial path condition. The next two parameters are the initial symbolic state and the set of locations written on the path - both are initially empty. $d$ is the bound on the length of the path condition that will be explored in producing the summary.

To produce a symbolic summary for the $k$-way interaction, we now compose $i_{\text {sum }}, f_{\text {sum }}$, and the edge summary computed by eSum(). There are two cases to consider. If the feature, $f^{\prime}$, is connected to $\operatorname{root}(i)$ with an edge, $\left(\operatorname{root}(i), f^{\prime}\right)$ we compose summaries in the following order: $i_{\text {sum }},\left(\operatorname{root}(i), f^{\prime}\right)_{\text {sum }}, f_{\text {sum }}^{\prime}$. If the feature, $f^{\prime}$, is connected to a leaf of $i, l_{i}$, with an edge, $\left(f^{\prime}, l_{i}\right)$ we compose summaries in the following order: $f_{\text {sum }}^{\prime},\left(f^{\prime}, l_{i}\right)_{\text {sum }}, i_{\text {sum }}$. 
Order matters in composing summaries because the set of written locations of two summaries may overlap and simply conjoining the equality constraints on the values at such locations will likely result in constraints that are unsatisfiable. We keep only last write of locations in a composed summary to honor the sequencing of writes and reads of locations that arise due to the order of composition.

Consider the composition of summary $s$ with summary $s^{\prime}$, in that order. Let $(p c, w) \in s$ and $\left(p c^{\prime}, w^{\prime}\right) \in s^{\prime}$ be two elements of those summaries. The concern is that $\operatorname{dom}(w) \cap \operatorname{dom}\left(w^{\prime}\right) \neq \emptyset$, where $\operatorname{dom}()$ extracts the set of locations used to index into a map. Our goal is to eliminate the constraints in $w$ on locations in $\operatorname{dom}(w) \cap \operatorname{dom}\left(w^{\prime}\right)$. In general, $p c^{\prime}$ will read the value of at least one location, $l$, and that location may have been written by the preceding summary. In such a case, the input value referenced in $p c^{\prime}$ should be equated to $w(l)$. Algorithm 2(right) composes two summaries taking care of these two issues.

In our approach, the generation of a symbolic summary produces "fresh" symbolic variables to name the values of inputs. A map, input(), records the relationship between input locations and those variables. We write $\operatorname{input}(s, l)$ to denote a summary $s$ and a location $l$ to access the symbolic variable. For a given path condition, $p c$, a call to $\operatorname{read}(p c)$ returns the set of locations referenced in the constraint - it does this by mapping back from symbolic variables to the associated input locations. We rely on these utility functions in Algorithm 2(right).

The algorithm considers all pairs of summary elements and generates, through the analysis of the locations that are written by the first summary and read by the second summary, a set of equality constraints that encode the path condition of the second summary element in terms of the inputs of the first. The pair of path conditions along with these equality constraints are checked for satisfiability. If they are satisfiable, then the cumulative write effects of the summary composition are constructed. All of the writes of the later summary are enforced and the writes in the first that are shadowed by the second are eliminated which eliminates the possibility of false inconsistency.

\subsection{Complexity and Optimization of Summary Composition}

From studying the Algorithm 2 it is apparent that the worst-case cost of constructing all summaries up to $k$-way summaries is exponential in $k$. This is due to the quadratic nature of the composition algorithm.

In practice we see quite a different story, in large part because we have optimized summary composition significantly. First, when we can determine that a pair of elements from a summary that might potentially match we ensure that for any shared features the summaries agree on the values for the elements of those summaries; this can be achieved through a string comparison of the summary constraints which is much less expensive than calling the SAT solver. Second, we can efficiently scan for constraints in one summary that are not involved in another summary and those can be eliminated since they were already found to be satisfiable in previous summary analyses. 


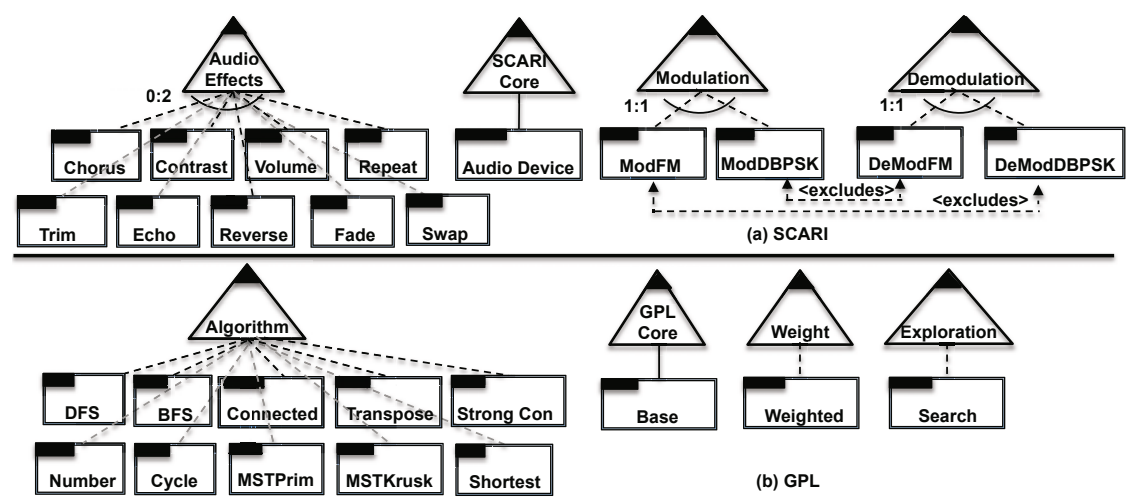

Fig. 2. Feature Models for (top) SCARI and (bottom) GPL

\section{Case Study}

We have designed a case study for evaluating the feasibility of our approach that ask the following two research questions. (RQ1): What is the reduction from our dependency analysis on the number of interactions that should be tested in an SPL? (RQ2): What is the difference in time between using our compositional symbolic technique versus a traditional directed technique?

\subsection{Objects of Analysis}

We selected two software product lines. The first SPL is based on the implementation of the Software Communication Architecture-Reference Implementation (SCARI-Open v2.2) [4] and the second is a graph product line, GPL [1, 14] used in several other papers on SPL testing.

The first product line, SCARI, was constructed by us as follows. First we began with the Java implementation of the framework. We removed the nonessential part of the product line (e.g. logging, product installation and launching) and features that required CORBA Libraries to execute. We kept the core mandatory feature, Audio Device, and transformed four features that were written in C (ModFM, DemodFM, Chorus and Echo), into Java. We then added 9 other features which we translated from $\mathrm{C}$ to Java from the GNU Open Source Radio [7] and the Sound Exchange (SoX), site 22. Table 1 shows the origin of each feature and the number of summaries for each. We used the example function for assembling features, to write a configuration program that composes the features together into products. The feature model is shown in Figure 2(a).

The graph product line (GPL) 14 has been used for various studies on SPLs. We start with the version found in the implementation site for [11. To fit our prototype tool, we re-factored some code so that every feature is contained in a method. We removed several features because either we could not find a method in the source code or because JPF would not run. We made the method Prog 
Table 1. SCARI Size by Feature

\begin{tabular}{|l|l|c|c|c|}
\hline Features & Origin & LOC & No. Summaries \\
\hline Chorus & 4 & 30 & 6 \\
\hline Contrast & 22 & 14 & 5 \\
\hline Volume & 22 & 47 & 5 \\
\hline Repeat & 22 & 12 & 3 \\
\hline Trim & 22 & 11 & 6 \\
\hline Echo & 4 & 31 & 5 \\
\hline Reverse & 22 & 14 & 4 \\
\hline Fade & $\mathbf{2 2}$ & 9 & 4 \\
\hline Swap & 22 & 27 & 4 \\
\hline AudioDevice & 4 & 13 & 3 \\
\hline ModFM & 4 & 19 & 4 \\
\hline ModDBPSK & 7 & 6 & 2 \\
\hline DemodFM & 4 & 18 & 4 \\
\hline DemodDBPSK & 7 & 6 & 3 \\
\hline \hline Total & & $\mathbf{2 5 7}$ & $\mathbf{5 8}$ \\
\hline
\end{tabular}

Table 2. GPL Size by Feature

\begin{tabular}{|l|c|c|}
\hline Features & LOC & No. Summaries \\
\hline Base & 85 & 56 \\
\hline Weighted & 32 & 148 \\
\hline Search & 35 & 19 \\
\hline DFS & 23 & 41 \\
\hline BFS & 23 & 6 \\
\hline Connected & 4 & 8 \\
\hline Transpose & 27 & 3 \\
\hline StronglyConnected & 19 & 9 \\
\hline Number & 2 & 2 \\
\hline Cycle & 40 & 19 \\
\hline MSTPrim & 92 & 4 \\
\hline MSTKruskal & 106 & 3 \\
\hline Shortest & 102 & 3 \\
\hline Total & $\mathbf{5 9 0}$ & $\mathbf{3 2 1}$ \\
\hline
\end{tabular}

our main entry point for the program. We did not include any constraints for simplicity. Figure 2(b) shows the resulting feature model and Table 2 shows the number of lines of code and the number of summaries by feature.

\subsection{Method and Metrics}

Experiments are run on an AMD Linux computing cluster running CentOS 5.3 with 128GB memory per node. We use Java Pathfinder (JPF) [15] to perform SE with the Choco solver for SCARI and CVC3BitVector for GPL. We adapt the information flow analysis (IFA) package [9] in Soot 25] for our FDG. In SCARI we use the configuration program for a starting point of analysis. In GPL we use the Prog program, which is an under-approximation of the FDG.

For RQ1 we compute the number of possible interactions (directed and undirected) at increasing values for $k$, obtained directly from the feature model. We compare this with the number that we get from the interaction trees. For RQ2, we compare the time that is required to execute the two symbolic techniques on all of the trees for increasing values of $k$. We compare incremental SE (IncComp) and a full direct SE (DirectSE). We set the depth for SE at 20 for IncComp and allow DirectSE $k$-times that depth since it works on the full partial-product each time, while IncComp composes $k$ summaries each computed at depth 20. DirectSE does not use summaries, but in the SPLs we studied there is no opportunity for summary reuse within the analysis of a partial product our technique reuses summaries across partial products.

\subsection{Results}

RQ1. Table 3 compares the number of interactions obtained from just the OVM with the number of interaction trees obtained through our dependency analysis. We present $k$ from 2 to 5 . The column labelled UI is the number of interactions calculated from all $k$-way combinations of features. In SCARI there are only three true points of variation given the model and constraints, therefore we see the same number of interactions for $k=3$ and 4 . For $k=5$, we have fewer interactions since there are 5 unique 4-way feature combinations in a single product with 5 features, but only a single 5 -way combination. The DI column 
Table 3. Reduction for Undirected (U) and Directed (D) Interactions (I)

\begin{tabular}{|c|c|c|c|c|c|c|c|}
\hline Subject & UI & DI & Feasible UI & Feasible DI & UI Reduction & DI Reduction \\
\hline \multirow{3}{*}{ SCARI } & 2 & 188 & 376 & 85 & 85 & $54.8 \%$ & $77.4 \%$ \\
\cline { 2 - 8 } & 3 & 532 & 3192 & 92 & 92 & $82.7 \%$ & $97.1 \%$ \\
\cline { 2 - 7 } & 4 & 532 & 12768 & 162 & 162 & $69.5 \%$ & $98.7 \%$ \\
\cline { 2 - 7 } & 5 & 164 & 19680 & 144 & 144 & $12.2 \%$ & $99.3 \%$ \\
\hline \multirow{3}{*}{ GPL } & 2 & 288 & 576 & 21 & 27 & $92.7 \%$ & $95.3 \%$ \\
\cline { 2 - 7 } & 3 & 2024 & 12144 & 29 & 84 & $98.6 \%$ & $99.3 \%$ \\
\cline { 2 - 7 } & 4 & 9680 & 232320 & 31 & 260 & $99.7 \%$ & $99.9 \%$ \\
\cline { 2 - 7 } & 5 & 33264 & 3991680 & 20 & 525 & $99.9 \%$ & $100.0 \%$ \\
\hline
\end{tabular}

represents the number of directed interactions or all permutations $(k ! \times U I)$. The next two columns are feasible interactions obtained from the interaction trees. Feasible UI, removes direction, counting all trees with the same features as equivalent. Feasible DI is the full tree count. The last two columns give the percent reduction. For the undirected interactions we see a reduction of between $12.2 \%$ and $99.9 \%$ across subjects and values of $k$, and the reduction is more dramatic in GPL (92.7\%-99.9\%). If we consider the directed interactions, which would be needed for test generation, there is a reduction ranging from $77.4 \%$ to $100 \%$. In terms of absolute values we see a reduction in GPL from over 3 million directed interactions at $k=5$, down to 525, an order 4 magnitude of difference. DIs are useful to detect more behaviors. For example, given a onesecond-sound file, trim $\rightarrow$ repeat removes 1-second-sound and generates an empty file; repeat $\rightarrow$ trim repeats the sound once and outputs a 1-second-sound file.

RQ2. Table 4 compares the performance of DirectSE and IncComp in terms of time (in seconds). It lists the number of directed (D) and undirected (U) interactions (I) for each $k$, that are feasible based on the interaction trees. Some features in the feature models may have more than one method. In RQ1 based on the OVM we reported interactions only at the feature level. However in this table, we consider all methods within a feature and give a more precise count of the interactions; we list all of the interactions (both directed and undirected) between features. The next two columns present time. For Direct SE we re-start the process for each $k$, but for the IncComp technique we use cumulative times because we must first complete $k-1$ to compute $k$. Although both techniques use the same time for single feature summaries, they begin to diverge quickly. DirectSE is 3 times slower for $k=5$ on SCARI, and 4 times slower on GPL. Within SCARI we see no more than a 3 second increase to compute $k+1$ from $k$ (compared to 14-35 seconds for DirectSE) and in GPL we see at most 750 (12 mins). For DirectSE it requires as long as 3160 (about 1 hour).

The last column of this table shows how many feasible paths were sent to the SAT solver (SAT). We saw (but don't report) a similar number for DirectSE which we attribute to our depth bounding heuristic. The number for SMT represents the total number of possible calls that were made to the SAT solver. However, we did not send all possible calls, because our matching heuristic culled out a number which we show as Avoided Calls. 
Table 4. Time Comparisons for SCARI and GPL

\begin{tabular}{|c|c|c|c|c|c|c|}
\hline \multirow{5}{*}{ Subject } & & Feasible UI & Feasible DI & DirectSE & \multicolumn{2}{c|}{ IncComp } \\
\cline { 2 - 6 } & & & & Time (sec) & Time (sec) & SAT/SMT, Avoided Calls \\
& 1 & 14 & 14 & 6.75 & 6.75 & 58 \\
\cline { 2 - 6 } & 2 & 85 & 85 & 14.48 & 9.63 & $430 / 1780,0$ \\
\cline { 2 - 7 } & 3 & 92 & 92 & 17.67 & 10.06 & $844 / 2226,1587$ \\
\cline { 2 - 7 } & 4 & 162 & 162 & 36.09 & 10.93 & $1505 / 2909,3442$ \\
\cline { 2 - 7 } & 5 & 144 & 144 & 35.87 & 11.70 & $2075 / 3523,5696$ \\
\hline \hline \multirow{3}{*}{ GPL } & 1 & 49 & 49 & 41.77 & 41.77 & 321 \\
\cline { 2 - 7 } & 2 & 60 & 76 & 67.25 & 56.28 & $663 / 985,0$ \\
\cline { 2 - 7 } & 3 & 81 & 310 & 184.76 & 82.00 & $1441 / 1901,1809$ \\
\cline { 2 - 7 } & 4 & 82 & 1725 & 727.34 & 216.63 & $5814 / 7342,5396$ \\
\cline { 2 - 7 } & 5 & 52 & 8135 & 3887.23 & 965.92 & $27444 / 34147,19743$ \\
\hline
\end{tabular}

\section{Conclusions and Future Work}

In this paper we have presented a compositional symbolic execution technique for integration testing of software product lines. Using interaction trees to guide incremental summary composition we can efficiently account for all possible interactions between features. We consider interactions as directed which gives us a more precise notion of interaction than previous research. In a feasibility study we have shown that we can (1) reduce the number of interactions to be tested by a factor of between $12.2 \%$ and $99.9 \%$ over an uninformed model, and (2) reduce the time taken to perform symbolic execution by as much as factor of 4 over a directed symbolic execution technique. Another advantage of this technique is that since our results and costs are cumulative, we can keep increasing $k$ as time allows, making our testing stronger, without any extraneous work along the way.

As future work we plan to exploit the information gained from our analysis to perform directed test generation. By using the complete paths we can generate test cases from the constraints that can be used with more refined oracles. For paths which reach the depth bound, we plan to explore ways to characterize these partial paths to guide other forms of testing, such as random testing, to explore the behavior which is otherwise unknown.

Acknowledgements. This work is supported in part by the National Science Foundation through awards CCF-0747009 and CCF-0915526, the Air Force Office of Scientific Research through awards FA9550-09-1-0129 and FA9550-101-0406, and the National Aeronautics and Space Administration under grant number NNX08AV20A.

\section{References}

1. Bertolino, A., Gnesi, S.: PLUTO: A Test Methodology for Product Families. In: van der Linden, F.J. (ed.) PFE 2003. LNCS, vol. 3014, pp. 181-197. Springer, Heidelberg (2004)

2. Cohen, M.B., Colbourn, C.J., Gibbons, P.B., Mugridge, W.B.: Constructing test suites for interaction testing. In: Proc. of the Intl. Conf. on Soft. Eng., pp. 38-48 (May 2003)

3. Cohen, M.B., Dwyer, M.B., Shi, J.: Coverage and adequacy in software product line testing. In: Proc. of the Workshop on the Role of Arch. for Test. and Anal., pp. 53-63 (July 2006) 
4. Communication Research Center Canada, http://www.crc.gc.ca/en/html/crc/ home/research/satcom/rars/sdr/products/scari_open/scari_open

5. de Moura, L., Bjørner, N.S.: Z3: An Efficient SMT Solver. In: Ramakrishnan, C.R., Rehof, J. (eds.) TACAS 2008. LNCS, vol. 4963, pp. 337-340. Springer, Heidelberg (2008)

6. Geppert, B., Li, J., Rößler, F., Weiss, D.M.: Towards Generating Acceptance Tests for Product Lines. In: Dannenberg, R.B., Krueger, C. (eds.) ICSR 2004. LNCS, vol. 3107, pp. 35-48. Springer, Heidelberg (2004)

7. GNU Radio, http://gnuradio.org/redmine/wiki/gnuradio

8. Godefroid, P.: Compositional dynamic test generation. In: Proc. of the ACM Symposium on Principles of Programming Languages, pp. 47-54 (2007)

9. Halpert, R.L.: Static lock allocation. Master's thesis, McGill University(April 2008)

10. Jaring, M., Bosch, J.: Expressing product diversification - categorizing and classifying variability in software product family engineering. Intl. Journal of Soft. Eng. and Knowledge Eng. 14(5), 449-470 (2004)

11. Kim, C.H.P., Batory, D., Khurshid, S.: Reducing combinatorics in testing product lines. In: Asp. Orient. Soft. Dev., AOSD (2011)

12. King, J.C.: Symbolic execution and program testing. Commun. ACM 19(7), 385-394 (1976)

13. Kuhn, D., Wallace, D.R., Gallo, A.M.: Software fault interactions and implications for software testing. IEEE Trans. on Soft. Eng. 30(6), 418-421 (2004)

14. Lopez-Herrejon, R.E., Batory, D.: A Standard Problem for Evaluating ProductLine Methodologies. In: Dannenberg, R.B. (ed.) GCSE 2001. LNCS, vol. 2186, pp. 10-24. Springer, Heidelberg (2001)

15. NASA Ames. Java Pathfinder (2011), http://babelfish.arc.nasa.gov/trac/jpf

16. Ottenstein, K.J., Ottenstein, L.M.: The program dependence graph in a software development environment. In: Proc. of the Soft. Eng. Symp. on Practical Soft. Develop. Envs., pp. 177-184 (1984)

17. Person, S., Dwyer, M.B., Elbaum, S., Păsăreanu, C.S.: Differential symbolic execution. In: Intl. Symp. on Foun. of Soft. Eng., pp. 226-237 (2008)

18. Pohl, K., Böckle, G., van der Linden, F.J.: Software Product Line Engineering: Foundations, Principles and Techniques. Springer, Heidelberg (2005)

19. Reis, S., Metzger, A., Pohl, K.: Integration Testing in Software Product Line Engineering: A Model-Based Technique. In: Dwyer, M.B., Lopes, A. (eds.) FASE 2007. LNCS, vol. 4422, pp. 321-335. Springer, Heidelberg (2007)

20. Reisner, E., Song, C., Ma, K.-K., Foster, J.S., Porter, A.: Using symbolic evaluation to understand behavior in configurable software systems. In: Intl. Conf. on Soft. Eng., pp. 445-454 (May 2010)

21. Reps, T., Rosay, G.: Precise interprocedural chopping. In: Proc. of the ACM Symposium on Foundations of Soft. Eng., pp. 41-52 (1995)

22. Sox. Sound Exchange (2011), http://sox.sourceforge.net/

23. Stricker, V., Metzger, A., Pohl, K.: Avoiding Redundant Testing in Application Engineering. In: Bosch, J., Lee, J. (eds.) SPLC 2010. LNCS, vol. 6287, pp. 226-240. Springer, Heidelberg (2010)

24. Uzuncaova, E., Garcia, D., Khurshid, S., Batory, D.: Testing software product lines using incremental test generation. In: Intl. Symp. on Soft. Reliab. Eng, pp. 249-258 (2008)

25. Vallée-Rai, R., Gagnon, E.M., Hendren, L., Lam, P., Pominville, P., Sundaresan, V.: Optimizing Java Bytecode Using the Soot Framework: Is It Feasible? In: Watt, D.A. (ed.) CC 2000. LNCS, vol. 1781, pp. 18-34. Springer, Heidelberg (2000) 